\section{1,2,3,5,8,8a-Hexahydronaphthalin aus 1,2-Cyclohexadien * *}

\section{Von Manfred Christl* und Michael Schreck}

Obwohl experimentelle und theoretische Ergebnisse die reaktive Zwischenstufe 1,2-Cyclohexadien 1a sehr gut charakterisieren ${ }^{\text {(I) }}$, sind Anwendungen von 1a in der Synthese bisher nicht beschrieben. Angeregt durch die schon unter milden Bedingungen erfolgende Umlagerung ${ }^{[2 u]}$ des $[2+2]$ Cycloaddukts von 1,3-Butadien an Tricyclo[4.1.0.0 $\left.{ }^{2.7}\right]$ hepta-3,4-dien ${ }^{[2 \mathrm{~b}]}$, haben wir jetzt die Reaktionen von 1,3-Butadien und einigen seiner Methylderivate mit la und 1Methyl-1,2-cyclohexadien $\mathbf{1 b}^{[3]}$ sowie den Übergang der $[2+2]$-Cycloaddukte 2 und 3 in das bisher unbekannte $1,2,3,5,8,8 \mathrm{a}-\mathrm{Hexahydronaphthalin} \mathbf{4 a}$ und einige seiner Methylderivate studiert.

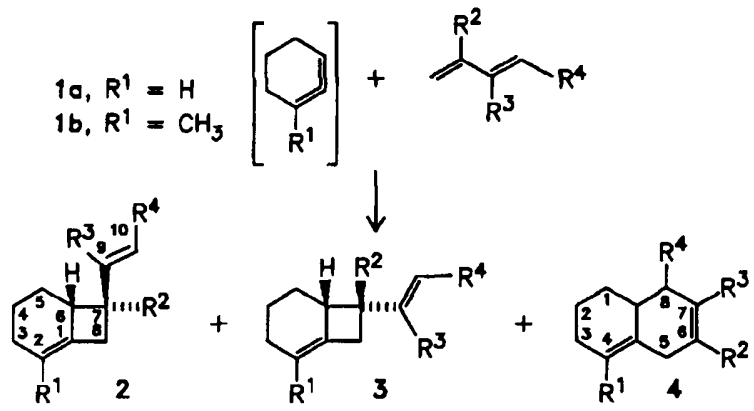

a, $R^{1}=R^{2}=R^{3}=R^{4}=H ; b, R^{1}=R^{3}=R^{4}=H, R^{2}=C_{3} ;$ c, $R^{1}=R^{2}=R^{4}=H, R^{3}=C_{3} ; d, R^{1}=R^{2}=R^{3}=H, R^{4}=C_{3} ;$ •. $R^{1}=R^{4}=H, R^{2}=R^{3}=C_{3} ; f, R^{1}=C_{3}, R^{2}=R^{3}=R^{4}=H$

Schema 1 [6].

Bottini et al. ${ }^{[4]}$ hatten aus $1 \mathrm{a}$ und cis-1,3-Pentadien, trans,trans-2,4-Hexadien sowie 2,3-Dimethylbutadien 7-Vinylbicyclo[4.2.0]oct-1-ene des Typs 2. und 3 erhalten, aber keine Konfiguration der Produkte angegeben. Wir setzten nun 1a ebenfalls durch Behandlung von 6,6-Dibrombicyclo[3.1.0]hexan mit Methyllithium ${ }^{[5]}$ frei und erhielten bei -20 bis $-15^{\circ} \mathrm{C}$ mit 1,3-Butadien, trans-1,3-Pentadien, Isopren und 2,3-Dimethylbutadien, die jeweils als LOsungsmittel dienten, die $[2+2]$-Cycloaddukte 2 und 3 sowie teilweise auch die [4+2]-Cycloaddukte 4 (Schema 1). Analog lieferte $\mathbf{1 b}^{[3 \mid}$ mit 1,3-Butadien die Produkte 2f, if und 5.

Tabelle 1. Ausbeuten [\%] an 2-4 und 5 nach gaschromatographischer Reinigung.

\begin{tabular}{lrrrlll}
\hline & & b & c & d & c & 1 \\
\hline 2 & 31 & 27 & 5 & 17 & 42 & 29 \\
4 & 4 & 5 & 3 & & & $1+5(2)$ \\
\hline
\end{tabular}

Die Verbindungen 2-5 wurden durch präparative Gaschromatographie (Ethylenglykoladipat-Säule, ca. $100^{\circ} \mathrm{C}$ ) gereinigt. In Tabelle 1 sind die Ausbeuten zusammengefaßt. Tabelle 2 enthălt ausgewāhlte NMR-spektroskopische Daten.

[*] Prof. Dr. M. Christl, Dr. M. Schreck

Institut für Organische Chemie der Universităt

Am Hubland, D-8700 Wurzburg

[*) Diese Arbeit wurde vom Fonds der Chemischen Industrie gefordert.
Besonders genau untersuchten wir die Reaktionen von 1a mit 1,3-Butadien und Isopren. Dabei zeigte sich, daß 4a und daher wohl auch seine Methylderivate Primäraddukte sind und nicht Folgeprodukte von 2 oder 3 (siehe unten). Aus dem Verhăltnis $(2 b+3 b): 2 c=6.4$ folgt, daB la die höher substituierte Doppelbindung von Isopren bevorzugt angreift. In einem Konkurrenzexperiment ermittelten wir das Verhältnis der Geschwindigkeitskonstanten der Reaktionen von 1,3-Butadien und 2,3-Dimethylbutadien mit 1a zu 1.9; dies bedeutet, daB 1,3-Butadien zu den reaktiveren Abfangreagentien für $1 \mathbf{a}^{[4]} z$ ăhlt.

Als Mechanismus für die Bildung von 2 und 3 liegt ein zweistufiger Prozeß nahe, wie er für die Addition von Styrol an 1a bereits bewiesen ist $t^{[3,7]}$. Wahrscheinlich reagieren 1a und 1,3-Butadien zum Diradikal 6 mit trans-konfigurierter Allylgruppe in der Seitenkette, das dann zu 22 oder 3a cyclisiert. Das Diradikal $7 \mathrm{mit}$ cis-konfigurierter Allylgruppe kommt als Vorstufe des [4+2]-Cycloaddukts $4 a$ in Frage, falls 7 direkt aus 1a und 1,3-Butadien hervorgehen sollte.

$$
\begin{aligned}
& 20,30 \stackrel{140^{\circ} \mathrm{C}}{\longrightarrow} 40(89 \%) \quad 20 \stackrel{150^{\circ} \mathrm{C}}{\longrightarrow} 4 d(68 \%) \\
& 2 b, 3 b \stackrel{140^{\circ} \mathrm{C}}{\longrightarrow} 4 b(74 \%) \quad 2 a \stackrel{170^{\circ} \mathrm{C}}{\longrightarrow} 40(86 \%) \\
& 2 c \stackrel{165^{\circ} \mathrm{C}}{\longrightarrow} 4 \mathrm{c}(68 \%)
\end{aligned}
$$

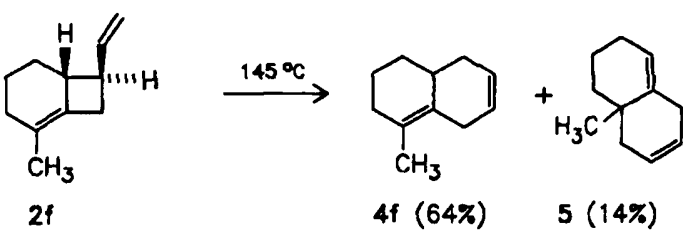

Schema $2|6|$.

Trotz der Bildung der Produktgemische ist die Addition von 1,3-Butadienen an 1a präparativ nutzlich, da diastereomere Bicyclo[4.2.0]oct-1-ene 2, 3 bei der Thermolyse in $\mathrm{C}_{6} \mathrm{D}_{6}$ zu einem $1,2,3,5,8,8 \mathrm{a}$-Hexahydronaphthalin 4 umlagern. Bei $140^{\circ} \mathrm{C}$ betrug die Halbwertszeit von $2 \mathrm{a}$ ca. $2 \mathrm{~h}$, die von $3 \mathrm{~b}$ ca. $18 \mathrm{~min}$. Schema 2 faßt diese Reaktionen und die Ausbeuten an isolierten Produkten zusammen. Somit sind das bisher unbekannte aa und einige seiner Methylderivate nunmehr leicht zugănglich.

$\mathrm{DaB}$ 4f aus $2 f$ entsteht, schließt für diesen Fall eine Cope-Umlagerung aus und macht sie für die anderen Ringerweiterungen $(2,3 \rightarrow \mathbf{4}, 2 \mathrm{f} \rightarrow 5)$ unwahrscheinlich. In Analogie zur Interpretation der Befunde bei den Thermolysen von 3-Vinylmethylencyclobutan und einigen seiner

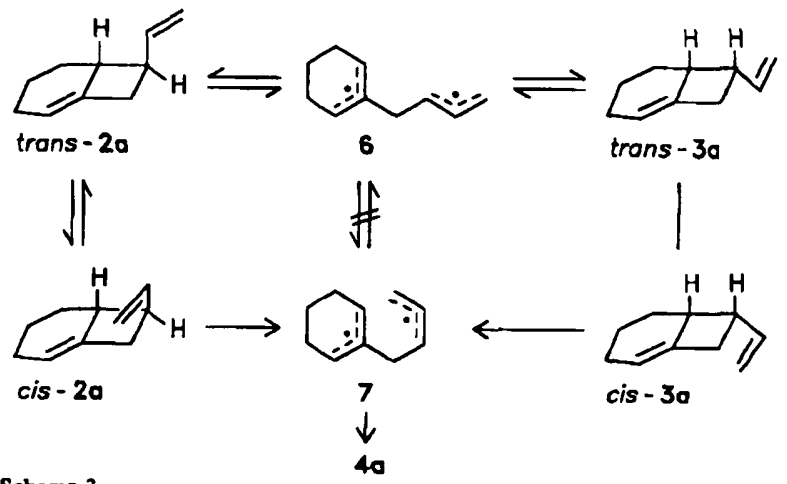

Schema 3. 
Derivate ${ }^{183}$ schlagen wir als Vorläufer von $4 a$ das Diradikal 7 vor, dessen Allylgruppe in der Seitenkette cis-konfiguriert ist. Wegen der Allylradikal-Rotationsbarriere von ca. $15 \mathrm{kcal} \mathrm{mol}^{-1[9]} \mathrm{kann} 7$ nicht direkt aus 6 hervorgehen, sondern nur aus cis-2a und cis-3a (Schema 3). Ein genaues Studium der Umlagerung von $2 \mathrm{a}\left(\Delta H^{+} \approx 33 \mathrm{kcal} \mathrm{mol}^{-1}\right.$, $\Delta S^{+} \approx 1 \mathrm{cal} \mathrm{mol}^{-1} \mathrm{~K}^{-1}$ ) und 3 a zeigte, daß parallel zur Ringerweiterung auch die Isomerisierung $2 a \rightleftharpoons 3 a$ ablăuft, wobei ausgehend von 2a der Anteil an 3a intermediăr maximal $1 \%$ und ausgehend von $3 \mathrm{a}$ jener an $2 \mathrm{a}$ maximal $7 \%$ betrug.

40

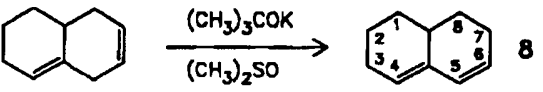

Von prăparativem Wert ist auch die baseinduzierte Isomerisierung von 4a. In $49 \%$ Ausbeute isolierten wir $1,2,3,7,8,8 \mathrm{a}-$ Hexahydronaphthalin 8 , das bisher nur in einer mehrstufigen Synthese als Gemisch mit einem Isomer ${ }^{[10]}$ erhalten worden war.

Tabelle 2. 'H- und ${ }^{13} \mathrm{C}$-NMR-Daten von $2 \mathrm{a}, 3 \mathrm{k}$, $4 a$ und $8\left(\mathrm{CDCl}_{3}, \delta\right.$-Werte, Kopplungskonstanten in $\mathrm{Hz}$ ).

2a : 'H-NMR [a]: $\delta=1.09\left(5-\mathrm{H}_{\text {eudn }}\right), 1.38\left(4-\mathrm{H}_{\text {eroo }}\right), 1.75\left(4-\mathrm{H}_{\text {endus }}\right), 1.91\left(5-\mathrm{H}_{\text {exo }}\right)$ $1.99\left(3-\mathrm{H}_{\text {endus }}\right), 2.08\left(3 \cdot \mathrm{H}_{\text {exo }}\right), 2.46(7-\mathrm{H}), 2.61\left(6-\mathrm{H}\right.$ und $\left.8-\mathrm{H}_{\text {exo }}\right), 2.70\left(8-\mathrm{H}_{\text {endudu }}\right)$ $4.89\left(\mathrm{dt}, J\left(7,10_{\text {trans }}\right)=J(10,10)=1.3, J\left(9,10_{\text {mams }}\right)=10.3 ; 10-H_{\text {moms }}\right), 4.95(\mathrm{dt}$, $\left.J\left(7,10_{\text {cis }}\right)=1.3, J\left(9,10_{\text {css }}\right)=17.3 ; 10-H_{\text {cis }}\right), 5.26(2-\mathrm{H}), 5.97$ (ddd, $J(7,9)=6.5$; 9-H). - ${ }^{13}$ C-NMR [b]: $\delta=21.54$ (C-4), 25.01 (C-3), 27.71 (C-5), 37.88 (C-8) 45.19 (C-7), 48.70 (C-6), 112.96 (C-10), 113.18 (C-2), 138.55 (C-1), 141.71 (C 9)

3a: 'H-NMR [a]: $\delta=1.16\left(5-\mathrm{H}_{\text {endu }}\right), 1.41\left(4-\mathrm{H}_{\text {exm }}\right), 1.52\left(5-\mathrm{H}_{\text {exo }}\right), 1.74\left(4-\mathrm{H}_{\text {endo }}\right)$, $1.99\left(3-\mathrm{H}_{\text {endo }}\right), 2.04\left(3-\mathrm{H}_{e \times n}\right), 2.30\left(8-\mathrm{H}_{\text {exo }}\right), 3.00\left(6-\mathrm{H}, 7-\mathrm{H}\right.$ und 8- $\left.\mathrm{H}_{\text {endo }}\right), 4.98-$ $5.04\left(\mathrm{~m} ; 10-\mathrm{H}_{\text {ris, }}, 10-\mathrm{H}_{\text {trams }}\right), 5.31(2-\mathrm{H}), 5.90(\mathrm{~m}, 9-\mathrm{H}),-{ }^{13} \mathrm{C}-\mathrm{NMR}$ [b]: $\delta=21.41(\mathrm{C}-4), 22.77$ (C-5), 24.82 (C-3), 36.87 (C-8), 40.67 (C-7), 45.58 (C-6), 114.12 (C-10), 114.71 (C-2), 138.55 (C-9), 140.18 (C-1)

4a: 'H-NMR: $\delta=1.37$ (dddd, $J=13.0,7.0,4.8,3.8$ ) und 1.84 (dddd, $J=13.0$, $9.8,6.5,3.5)\left(1-\mathrm{H}_{2}\right), 1.48-1.63\left(\mathrm{~m} ; 2-\mathrm{H}_{2}\right), 1.94(\mathrm{~m})$ und $2.12(\mathrm{br} \mathrm{dt}, J=17.2,5.3)$ $\left(8-\mathrm{H}_{2}\right), 1.99\left(\mathrm{~m} ; 3-\mathrm{H}_{2}\right), 2.29(\mathrm{~m} ; 82-\mathrm{H}), 2.58(\mathrm{dt}, J=20.0,3.7)$ und $2.86(\mathrm{dm}$, $J=20.0)\left(5-\mathrm{H}_{2}\right), 5.52(\mathrm{tt}, J=3.5,1.7 ; 4-\mathrm{H}), 5.57-5.70(\mathrm{~m} ; 6-\mathrm{H}, 7-\mathrm{H}) .{ }^{13} \mathrm{C}$. NMR [b]: $\delta=20.53(\mathrm{C}-2), 25.83$ (C-3), 29.63 (C-1), 33.53 (C-8a), 34.18 und 34.44 (C-5, C-8), 120.00 (C-4), 126.50 (Doppelbande; C-6, C-7), 137.16 (C. 4a)

8: 'H-NMR: $\delta=1.00-2.30(\mathrm{~m}$, starke Maxima bei 1.82 und 2.14; $11 \mathrm{H}$ ), 5.43 (m; 4-H), 5.65 (ddm, $J=9.9,5.0 ; 6-H), 5.98$ (dd, $J=9.9,2.0 ; 5-H$ ). $-{ }^{13} \mathrm{C}$. NMR: $\delta=22.68$ (C-2), 25.89 und 26.09 (C-3, C-7), 30.50 und 30.73 (C-1, C-8), 35.70 (C-8a), 123.51 (C-4), 127.12 und 129.59 (C-5, C-6), 137.52 (C-4a)

[a] Die Kopplungskonstanten zwischen den Protonen am Bicyclo[4.2.0]oct1-en-Gerust sind gleich groß wie jene in den 7-Arylderivaten [3]. [b] Bei der Zuordnung halfen off-resonance-entkoppelte oder protonengekoppelte Spektren sowie die Methylsubstituenteneffekte der Methylderivate.

Eingegangen am 23. Dezember 1986, verănderte Fassung am 23. Februar 1987 [Z 2026]

[1] Vgl. die Zitate in der Einleitung von [3].

[2] a) M. Christl, Angew. Chem. 93 (1981) 515; Angew. Chem. Int. Ed. Engl. 20 (1981) 529; R. Lang, Dissertation. Universităt Würzburg 1982; b) M. Christl, R. Lang, M. Lechner, Liebigs Ann. Chem. 1980, 980.

[3] M. Christl, M. Schreck, Chem. Ber. 120 (1987), im Druck.

[4] A. T. Bottini, L. L. Hilton, J. Plott, Tetrahedron 3] (1975) 1997.

[5] W. R. Moore, W. R. Moser, J. Org. Chem. 35 (1970) 908.

[6] Für alle Verbindungen liegen korrekte C-H-Analysenwerte, Massenspektren, ' $\mathrm{H}$ - und ${ }^{13} \mathrm{C}$-NMR-Spektren vor (siche auch Tabelle 2).

[7] S. Harnos, S. Tivakompannarai, E. E. Waali, Tetrahedron Lett. 27 (1986) 3701.

[8] D. Hasselmann, Tetrahedron Lelf. 1972, 3465; 1973, 3739; J. A. Berson, J. M. Janusz, J. Am. Chem. Soc. 96 (1974) 5939; W. R. Dolbier, Jr., G. J. Mancini, Tetrahedron Lett. 1975, 2141.

[9] R. Sustmann in H. G. Viehe, Z. Janousek, R. Merényi (Hrsg.): Substituent Effects in Radical Chemistry. Reidel, Dordrecht 1986, S. 143.

[10] H. Bohme, G. Peters, Z. Naturforsch. B 12 (1957) 5.

\section{EinfluB des H-Donors und der Temperatur auf die} Stereoselektivität von Radikalreaktionen ${ }^{\text {* }}$

Von Bernd Giese*, Juan Antonio González-Gómez, Stephen Lachhein und Jürgen O. Metzger*

Radikalreaktionen werden in steigendem Maße in der organischen Synthese eingesetzt, wobei die Kenntnis der Reaktivitäten und Selektivităten von entscheidender Bedeutung ist ${ }^{(1)}$. Eine besondere Rolle spielt die Stereoselektivităt, die wegen des frühen Übergangszustandes rascher Radikaireaktionen weniger von der Produktstabilităt, sondern mehr von der Abschirmung des Radikalzentrums abhängt. Dies wird wichtig bei $\mathrm{H}$-Abstraktionsreaktionen, weil die Ubertragung des kleinen $\mathrm{H}$-Atoms von der weniger abgeschirmten Seite zum thermodynamisch instabileren Produkt fuhrt. So liefert die Addition von Alkylradikalen an Methylmaleinsäureanhydrid $1 \mathrm{bzw}$. Phenylacetylen 3 im abschließenden $\mathrm{H}$-Übertragungsschritt durch Cyclohexylquecksilberhydrid bevorzugt die Isomere (Z)-2 bzw. (Z)-4, wobei die Selektivităt mit der Größe des Restes R zunimmt ${ }^{[2]}$.<smiles>CC1=CC(=O)OC1=O</smiles><smiles>C#Cc1ccccc1</smiles>

3<smiles>[R][C@H]1C(=O)OC(=O)[C@@H]1C</smiles>

(Z) -2<smiles>[R]/C=C\c1ccccc1</smiles>

(z) -4<smiles>[CH+]1CCCCC1</smiles><smiles>[R][C@H]1C(=O)OC(=O)[C@@H]1C</smiles>

(E) -2

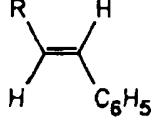

$(E)-4$

\begin{tabular}{|c|c|c|c|}
\hline $\mathbf{R}$ & $n-C_{6} H_{13}$ & $\begin{array}{l}b \\
c-C_{6} H_{11}\end{array}$ & $\begin{array}{l}\text { c } \\
t-\mathrm{C}_{4} \mathrm{H}_{9}\end{array}$ \\
\hline $\begin{array}{l}(Z)-2:(E)-2 \\
(Z)-4:(E)-4\end{array}$ & $62: 38$ & $\begin{array}{l}89: 11 \\
74: 26\end{array}$ & $\begin{array}{l}94: 6 \\
97: 3\end{array}$ \\
\hline
\end{tabular}

Am Beispiel des $\pi$-Vinylradikals $5 b^{[3]}$, gebildet durch Addition von Cyclohexylradikalen an Phenylacetylen, haben wir nun beobachtet, daß das $(Z) /(E)$-Verhăltnis auch durch Variation des H-Donors und der Reaktionstemperatur beeinflußt und sogar umgekehrt werden kann. Messungen zwischen -20 und $260^{\circ} \mathrm{C}$ lehren, daß $(Z)-4 b$ mit geringerer Aktivierungsenthalpie gebildet wird als $(E)-4 b$ (Tabelle 1).

Tabelle 1. Aktivierungsparameter für die Stereoselektivităt der H-Ćbertragung auf das Vinylradikal 5b.

\begin{tabular}{|c|c|c|c|}
\hline H-Donor & $\begin{array}{l}\Delta H^{+}((E) \cdot \Delta b)- \\
\Delta H^{+}((Z)-4 b) \\
{[k J / \mathrm{mol}]}\end{array}$ & $\begin{array}{l}\Delta S^{+}((E)-4 b)- \\
\Delta S^{+}((Z)-4 b) \\
{\left[J \mathrm{~mol}^{-1} \mathrm{~K}^{-1}\right]}\end{array}$ & $\begin{array}{l}\text { MeBtemp. } \\
{\left[{ }^{\circ} \mathrm{C}\right]}\end{array}$ \\
\hline $\begin{array}{l}c-\mathrm{C}_{6} \mathrm{H}_{11} \mathrm{HgH} \\
\mathrm{Bu}_{3} \mathrm{SnH} \\
c-\mathrm{C}_{6} \mathrm{H}_{12}\end{array}$ & $\begin{array}{r}2.5 \pm 0.2 \\
4.6 \pm 1.5 \\
11.7 \pm 1.0\end{array}$ & $\begin{array}{l}1.2 \pm 0.5 \\
7 \pm 5 \\
28 \pm 1.3\end{array}$ & $\begin{array}{r}-20-80 \\
0-84 \\
120-260\end{array}$ \\
\hline
\end{tabular}

[*] Prof. Dr. B. Giese, Dr. J. A. González-Gómez Dr. S. Lachhein Institut for Organische Chemie und Biochemie der Technischen Hochschule Petersenstraße 22, D.6100 Darmstadt

Priv.-Doz. Dr. J. O. Metzger Fachbereich Chemie der Universităt Carl-von-Ossietzky-Straße 9-11, D-2900 Oldenburg

[**] Diese Arbeit wurde von der Deutschen Forschungsgemeinschaft und dem Fonds der Chemischen Industrie gefordert. 